\title{
Rapid Prediction of Configuration Aerodynamics in the Conceptual Design Phase
}

C. Munro, P. Krus

\begin{abstract}
Conceptual aircraft design is characterised by the requirement to analyse a large number of configurations rapidly and cost effectively. For unusual configurations such as those typified by unmanned combat air vehicles (UCAVs) adequately predicting their aerodynamic characteristics through existing empirical methods is fraught with uncertainty. By utilising rapid and low cost experimental tools such as the water tunnel and subscale flight testing it is proposed that the required aerodynamic characteristics can rapidly be acquired with sufficient fidelity for the conceptual design phase. Furthermore, the initial design predictions can to some extent be validated using flight-derived aerodynamic data from subscale flight testing.
\end{abstract}

Keywords: aircraft design, UAV, water tunnel, flight testing.

\section{Introduction}

The conceptual design phase is characterised by the evaluation of many disparate concepts. Evaluation of these concepts requires the application of a combination of empirical, experimental and computational tools to predict the concept performance against requirements. However, this early design stage is also characterised by significant cost and time constraints. A significant level of risk must therefore be assumed because of insufficient time or financial resources to evaluate all the concepts comprehensively. In the case of configuration development for UCAVs there exists no empirical database and limited experience for the predicting of their aerodynamic characteristics. This is true not just because they are a recent concept in military aviation but also because other requirements, most prevalently low observability, are forcing extremely novel configurations. This tendency towards novel configuration solutions is also evident in the wide range of configurations emerging in the UAV field. The relevance of existing empirical tools and databases based on vastly different configurations is thus questionable.

It is argued in this paper that a quasi-physical modelling process is required to be able to truly capture the performance of the radically differing concepts typical of UCAVs. This approach attempts to combine the advantages of empirical, computational and experimental tools to enable the design team to better predict vehicle aerodynamic characteristics early in the design process with increased confidence, and hence to reduce risks.

\section{Background}

The interest in UCAVs has been driven by a number of factors, primarily the political necessity to minimise pilot casualties and to minimise cost at all levels. Principal amongst these is the drive for affordable weapon systems. It has been proposed, see for example [1], that UCAVs will lower acquisition, training and operational costs. Goals established in US programs in this area are for vehicle acquisition costs in the order of one-third the cost of the upcoming Joint Strike Fighter and have 50-80\% lower life cycle costs. These ambitious goals will be achieved through a number of means. Amongst these will be weight reduction (weight remains an important parameter in vehicle costs) gained from removing the pilot and associated equipment (such as cockpit heating, ejection seat and so forth) and training for the mission operators that can be conducted almost entirely in simulation (the vehicle need only fly on operational missions, hence eliminating fatigue and associated flight time driven cost drivers). The operational cost for a military operation would be minimised through maximising the platform survivability. It is this factor, and the demand for low observability - together with the freedom that accrues from removing the pilot from the vehicle - that has led to a large number of innovative, unconventional platforms. One example of this is the Saab SHARC concept, shown in Fig. 1.

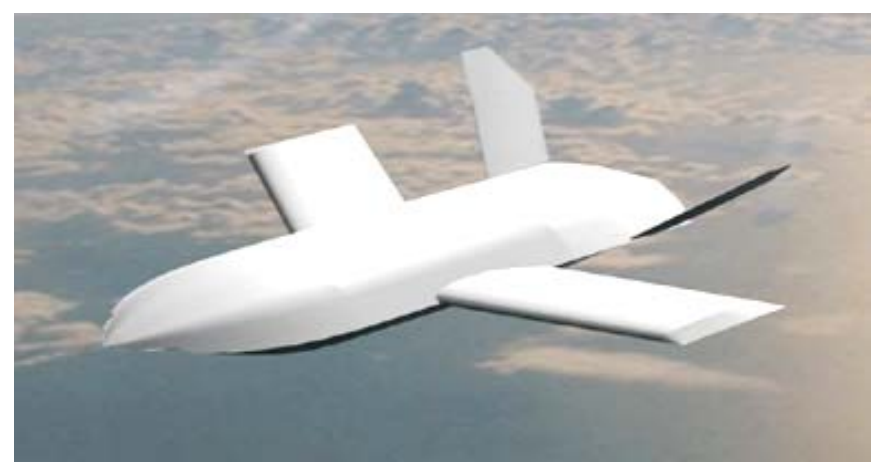

Fig. 1: Saab SHARC unmanned combat air vehicle concept (image courtesy Saab AB)

While there are undoubtedly many issues to be resolved with UCAVs regarding the level of autonomy, systems design, certification and interoperability with manned platforms, there also exist questions as to the aerodynamic performance of such vehicles. Advances in flight control certainly allow for a wide variation in vehicle design that would not otherwise be possible. However, the fundamental requirement still exists 
that the configuration must provide sufficient lift with low drag and the control surfaces be able to respond to demands to manoeuvre and ensure controllability. These aerodynamically derived characteristics are however rarely in concordance with low observability requirements.

The focus of the work described in this paper is to enable the aerodynamic design team to identify critical aerodynamic (primarily as related to control and stability) issues, predict performance and perform trade studies to determine the aerodynamic influence of changes (be they either to the external aerodynamics or to the control system). It should be recognised that there is very strong coupling in the case of UCAVs between the aerodynamics, stability and flight control functions and hence a need to be able to evaluate the interactions between the three early in the design process.

From a conceptual design standpoint, these requirements to meet both target performance and cost requirements place significant pressure on the initial analysis procedures. Furthermore, much of the cost (particularly that component driven by vehicle weight) is determined very early on, as illustrated in Fig. 2. Hence, there is significant leveraging and longer term payback that can be gained from investing earlier in conceptual design studies.

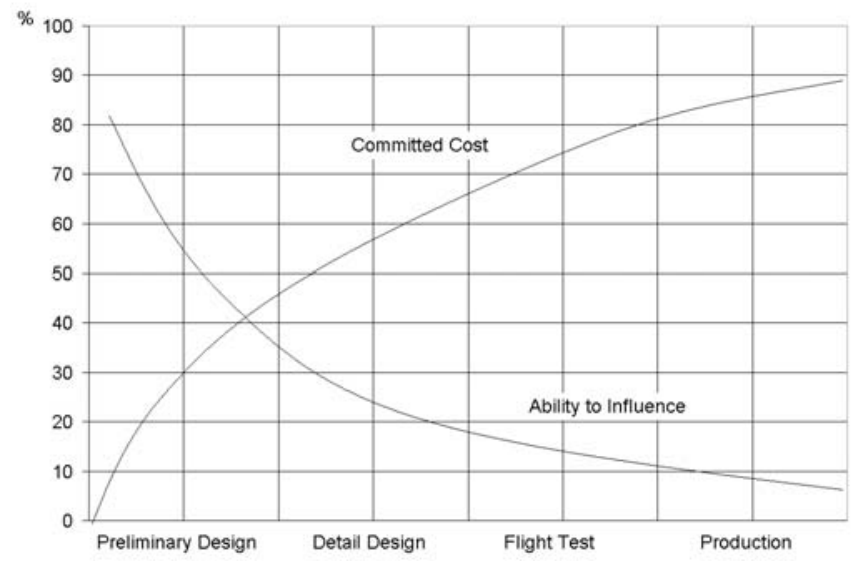

Fig. 2: Influence of design stage on costs and the ability to influence the design

The level of modelling fidelity is lower at the conceptual design stage out of necessity. There must therefore be an acceptable level of fidelity that is required to enable concept selection to be undertaken and the requirement definition to be refined. The methods presented in this paper attempt to address these combined requirements of low cost and time while achieving an acceptable level of fidelity for conceptual design prediction.

\section{Design analysis toolbox}

The use of computational tools for the prediction of flight vehicle performance is rightly assuming a great deal of importance in current aeronautical research. The benefits of such tools are clear: significantly reduced cost and time can, at least in principle, be achieved and often a greater insight into the underlying physics and multidisciplinary aspects can be obtained through the ability to exactly control test parameters which may well be coupled in the physical system.

In the field of flight performance the use of nonlinear six degree of freedom flight dynamics models is very well established as critical to every stage of vehicle development from initial system definition and design right through to training of aircrew. While the mathematical description of flight dynamics models is relatively simple and readily implementable in computer models, the same cannot be said for the underlying aerodynamics models. The complexity of fluids modelling means that computational fluid dynamics (CFD) is a relatively immature field that has not yet reached the point of acceptability in aircraft company conceptual design offices. The reasoning is generally that the cycle time is too long when there are so many configurations to be studied (and so many test points to run) and, often, a lack of confidence in the results.

To address this weakness in aerodynamic modelling at the conceptual design stage a method of building up the aerodynamic database using rapid experimental and computational tools around the central flight dynamics model is proposed, as shown in Fig. 3.

The role of the aerodynamic prediction methods presented is twofold: validation of simulation results and as a complement to elements of the digital simulation. From a set of designs sketched by the conceptual design team (with a large input from low observability elements as regards the

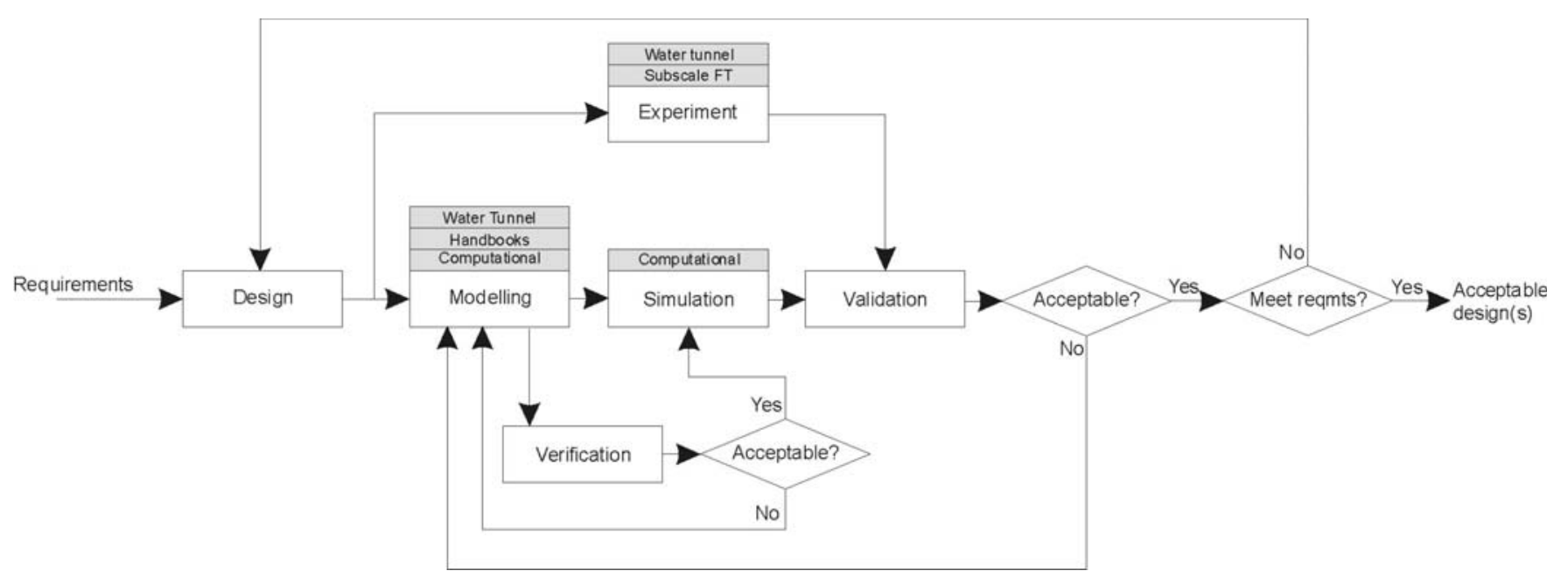

Fig. 3: Conceptual design process with physical and virtual modelling elements 
external configuration) a few may be selected on the basis of initial empirical analysis to proceed to more detailed modelling and experimental investigation. Such activities may proceed in parallel. Modelling will consist of initial computational analysis complemented by water tunnel quantitative data. This water tunnel data may be used to aid in generating the preliminary aerodynamic database that can be used as an input to the flight dynamics simulation model that can be used for initial operational/developmental evaluation. Furthermore, the flow visualisation capabilities of the water tunnel provide an insight into the aerodynamic behaviour and hence guide the design team to potential areas of improvement through a greater understanding of the dominant flow physics that this provides.

The terms verification and validation used here conform to the test and evaluation community definitions, as detailed in [2]. Verification need only be performed on major code changes, to ensure software robustness. Validation on the other hand is the confirmation of simulation results with experimental or other means. In this instance, the validation can be provided by the subscale flight test elements, providing an independent check of the modelling and simulation elements that are themselves a combination of experimental (water tunnel), empirical and digital simulation. The modelling can be adjusted on the basis of the validation to improve the prediction capability. The design team can thus have more confidence in the analysis results when it comes to evaluating these results against design requirements. This in turn increases the confidence in the design, helps identify key problem areas requiring further work and reduces the risk. Conversely, this reduced risk could be turned to allow more radical configurations to be evaluated with the same level of risk as without this approach.

Key elements of this design process are two relatively novel experimental tools: the water tunnel and subscale flight testing. It is the contention of this research that these methods both have significant potential in the conceptual design phase for UCAV aerodynamics, although their limitations are significant and must be recognised.

\section{Novel experimental tools}

While experimentation is undoubtedly a rather expensive and time consuming activity in comparison to simulation, the development of modern, cost effective and accurate instrumentation has driven down experimental costs and cycle time. Furthermore, questions remain in the conceptual design area over the feasibility in the near-term of utilising complex, long cycle time simulations. The water tunnel and subscale flight test tools that are introduced here are good examples of this trend. Both tools have their advantages and disadvantages in terms of time, cost and modelling fidelity, and these are introduced in this section.

\section{Water tunnel}

The water tunnel has long been used as a diagnostics tool for external flows (see for example [3] and [4]). For UCAVs, the demand for low observability tends to chined fuselage geometries and swept, relatively sharp wing leading edges. The flow over such geometries at moderate to high angles of attack will in general be dominated by vortical flow with fixed separation lines along the sharp wing leading edges and fuselage chines. This geometrically fixed separation combined with the vortex dominated separated flowfield makes such flows Reynolds insensitive ([5] and [6] are typical examples illustrating the Reynolds number insensitivity of such flows) and hence amenable to analysis in low Reynolds number facilities such as water tunnels.

While flow visualisation has been well demonstrated in water tunnels, the use of strain gauge balances to allow force and moment measurement is relatively new ([7], [8]). Initial test results performed as part of this work on generic delta wing models indicates excellent correlation of both lift curve slope and maximum lift coefficient with wind tunnel data, as shown in Fig. 4. Note that the Reynolds number as listed here is based on root chord and that the lift coefficient value does not account for the contribution of tangential force. Hence, the true lift coefficient is slightly lower at high angles of attack than shown here. Nonetheless, this result indicates the high level of accuracy that can be rapidly achieved while remaining within the time and cost constraints imposed at the conceptual design stage.

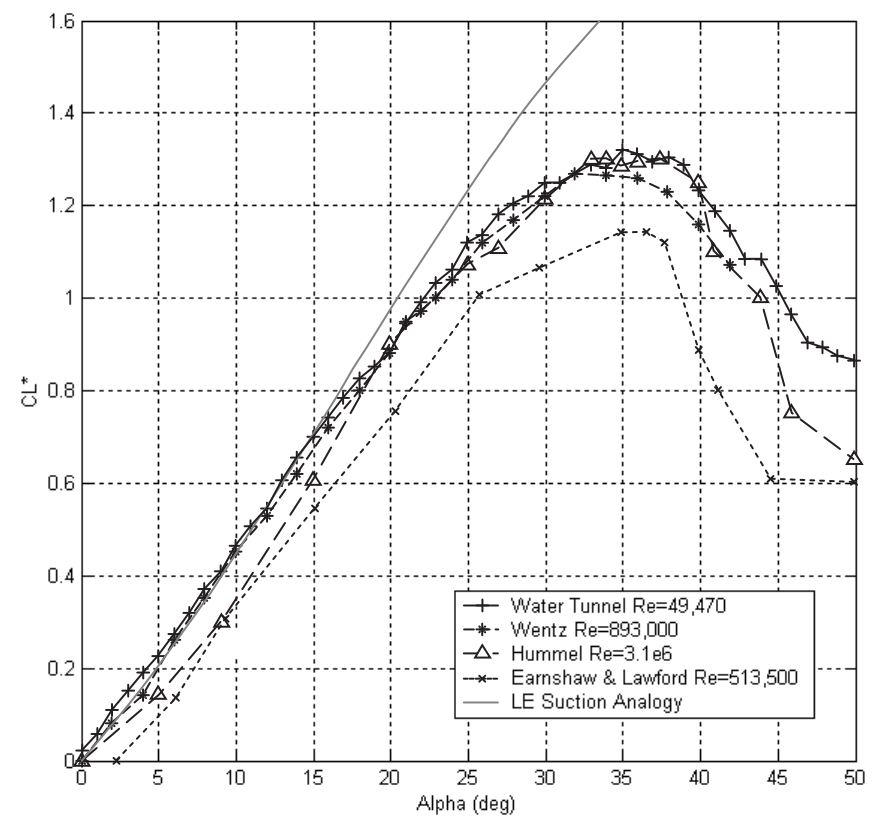

Fig. 4: Matching of force data for water and wind tunnel tests of a $65^{\circ}$ delta wing

\section{Subscale flight testing}

The use of subscale flying models for free dynamics testing has become increasingly possible in recent years due to the development of lightweight, low power instrumentation and telemetry systems. Examples of projects that have been undertaken in this area include the Boeing/NASA X-36 fighter $28 \%$ scale demonstrator [9], NASA experimental hypersonic configuration [10] and the Saab T28 instrumented model (see Fig. 5). 


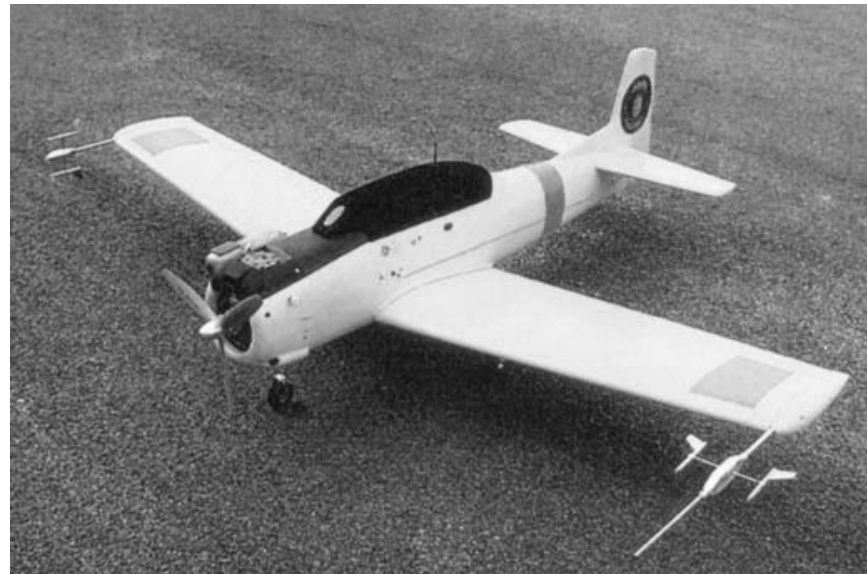

Fig. 5: T28 Trojan instrumented model aircraft

Subscale flight testing offers the benefit over ground-based tests of being fully six degrees of freedom, enabling realistic manoeuvres to be performed (although inertial scaling remains an issue requiring solution). Particularly for high risk tests such as at high angle of attack and departures such as spin, the subscale demonstrator offers the opportunity to explore these corners of the flight envelope in a safer manner than with a fullscale vehicle and at much lower cost. Furthermore, the subscale model can be built early in the design process (perhaps late in the conceptual phase) whereas the fullscale demonstrator can only be tested after design work is essentially complete. Particularly for UCAVs, these can readily demonstrate technologies key to unmanned operation in addition to exploring a configuration's aerodynamic performance.

Advances in parameter identification methods combined with the widespread availability of fast computers have enabled the rapid identification of aerodynamic parameters from flight test data. The resulting set of aerodynamic and control derivatives can then readily be compared to simulation results for validation and improvement of these prediction approaches. Furthermore, the possibility exists to perform tests rapidly with variations of platform geometry to assess alternative configurations and perform trade studies.

Initial testing has been performed to confirm that a complete set of inertial and air data can be readily recorded onboard and telemetered to the ground in real time for a payload weight of under $5 \mathrm{~kg}$ and at very low cost. Work is currently underway on a small UCAV-type vehicle for demonstration of the concept in a more realistic configuration. Further, the work will be validated against fullscale flight test and wind tunnel data on a number of representative configurations.

\section{Methods integration}

The two methods described in this paper are incorporated into the design process as shown in Fig. 3. As shown in Fig. 6, the two novel methods proposed here fit into a spectrum of experimental tools that can be utilised in the design process. Through careful selection of these methods with an underlying simulation capability that develops as the aircraft develops, the potential exists for more rapid and effective aerodynamic prediction. Note also how the tendency is for

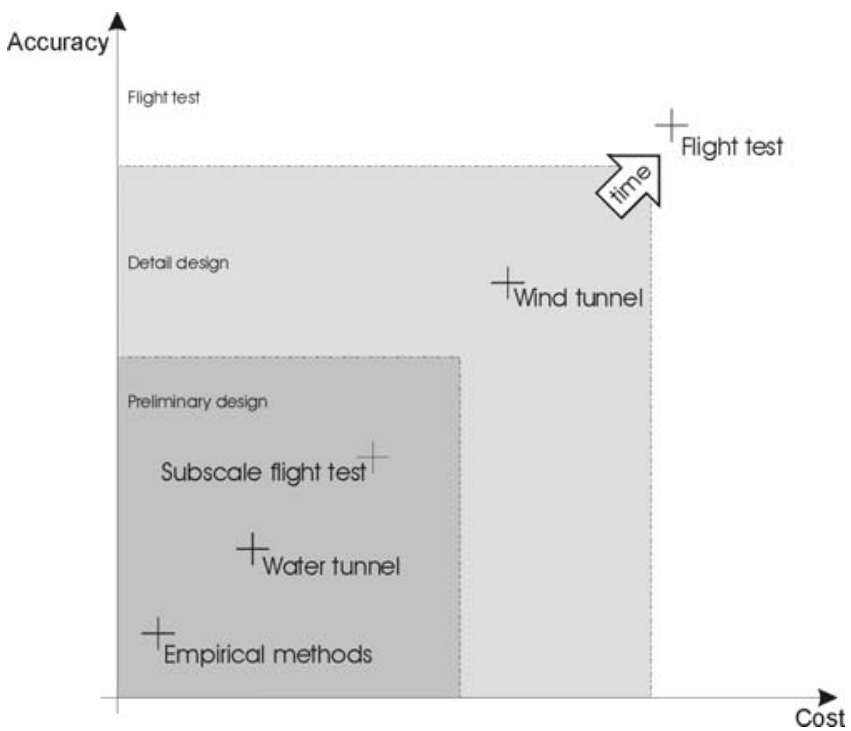

Fig. 6: Tradeoffs of accuracy and cost in methods selection

the last $10 \%$ of accuracy to cost much more than for the initial, conceptual design level estimate.

Neither water tunnel testing or subscale flight testing can replace the wind tunnel or fullscale flight testing in developing an aircraft to certification. However, at the very early design stages where there are a large number of options to be studied and time and financial resources are limited, these tools offer some potential to reduce risk and increase the design knowledge. This in turn, has a great leveraging effect on the overall development cost, as was indicated in Fig. 2.

\section{Conclusion}

The aerodynamic conceptual design of novel configurations such as UCAVs present significant problems to the aerodynamic designer, who is dependent on empirical (handbook) approaches combined with time consuming and costly wind tunnel and CFD analysis. It is proposed in the present research that useful additions to the design team's analysis toolbox may be the water tunnel and subscale flight testing. These two experimental methods fit into a simulation framework by enabling validation of simulation results and by complimenting flight dynamics modelling by reducing the need for more costly aerodynamic prediction methods early in the design process.

Further work is currently underway to validate both the water tunnel and subscale flight testing against baseline test configurations for which there are readily available wind tunnel and fullscale flight test data. Work is also underway to address the dynamic scaling issues relevant to subscale flight testing and how they can be addressed.

\section{Acknowledgments}

The first author's work in this area is supported by the Swedish Engineering, Design, Research and Education Agenda (ENDREA). The authors also wish to acknowledge the support of Saab AB in conducting this work. 


\section{References}

[1] DARPA System Capability Document - Unmanned Combat Air Vehicle Operational System. http://www.darpa.mil/tto/ downloadables/UCAV/APPENDIX.doc, 1998

[2] Hall, D. H., Kilikauskas, M., Laack, D. K., Muessig, P. R., O'Neal, B., Richardson, C., Simecka, K., Stewart, W., Turner, S. W.: How to VV\&A without really trying.US Joint Accreditation Support Activity JTCG/AS-97-M-00 9, 1997

[3] Del Frate, J. H.: NASA Dryden Flow Visualization Facility. NASA TM-4631, 1995

[4] Malcolm, G. N., Nelson, R. C.: Comparison of Water and Wind Tunnel Flow Visualization Results on a Generic Fighter Configuration at High Angles of Attack. AIAA 87-2423, 1987

[5] Erickson, G.: Vortex Flow Correlation. AFWAL-TR-80$-3142,1981$

[6] Roos, F. W., Kegelman, J. T.: An Experimental Investigation of Sweep-Angle Influence on Delta-Wing Flows. AIAA 90-0383, 1990

[7] Suarez, C. J., Malcolm, G. N.: Water Tunnel Force and Moment Measurements on an F/A-18. AIAA 94-1802, 1994
[8] Cunningham, Jr., A. M., Bushlow, T., Mercer, J. R., Wilson, T. A., Schwoerke, S. N.: Small Scale Model Tests in Small Wind and Water Tunnels at High Incidence and Pitch Rates. AD-A208647, Vol. 1-3, 1989

[9] Walker, L. A.: Flight Testing the X-36 - The Test Pilot's Perspective. NASA CR-198058, 1997

[10] Budd, G. D., Gilman, R. L., Eichstedt, D.: Operational and Research Aspects of a Radio-Controlled Model Flight Test Program. Journal of Aircraft, 32, 1995, pp. 583-589

Cameron Munro, BEng(Aero), BBus phone: +4613285783

fax: +4613130414

e-mail: cammu@ikp.liu.se

Petter Krus, Ph.D.

phone: +46 13281792

fax: +4613130414

e-mail:petkr@ikp.liu.se

Department of Mechanical Engineering

Linköpings Universitet

58183 Linköping, Sweden 